\title{
Hybrid Structures for Piezoelectric Nanogenerators: Fabrication Methods, Energy Generation, and Self- Powered Applications
}

\author{
Nagamalleswara Rao Alluri, \\ Arunkumar Chanderashkear and Sang-Jae Kim \\ Additional information is available at the end of the chapter
}

http://dx.doi.org/10.5772/intechopen.74770

\begin{abstract}
Smart energy harvesting through the surrounding environment generates sufficient energy to drive the low-power consumption systems. It is the forthcoming revolution in smart (or self-powered) technology and results in abolishing the usage of complex batteries, external circuit components, and natural sources. To date, extensive fabrication methods, the growth of $\mathrm{ZnO}$ nanostructures on plastic substrates, and flexible piezoelectric polymer film-based devices were tested to improve the performance of piezoelectric nanogenerator (PNG) as a prominent energy-harnessing approach for the development of sustainable independent power sources. Still, PNG technology suffers from brittleness, leakage current issues, high electrical output generation, and long-term durability, which can be possible to control by the composite technology, that is, polymer/nanoparticles. The objective of this book chapter determines the rapid growth of multifunctional, flexible composite structures through various methods (e.g., ionotropic gelation method, groove technique, ultrasonication followed by solution-casting methods) for high output energy generation and self-powered sensor/system studies.
\end{abstract}

Keywords: piezoelectric nanogenerator, composite structures, self-powered sensors, $\mathrm{BaTiO}_{3}$ nanoparticles, polyvinylidene fluoride (PVDF)

\section{Introduction}

The contemporary society and technology directing toward the digitalization of the world increases the huge consumption of electrical energy. Over the decades, the major percentage 
amount of electrical energy is generated by the consumption of the natural sources like oil, gas, coal, and water. However, the natural sources are limited in supply, and the continuous energy generation using these sources creates global warming issues and pollution, which will effect on the lifespan of the human race. Moreover, the regeneration of the natural sources takes hundreds of the thousand years. The international energy agency (IEA) statistics depicts that the energy crisis is increasing day by day due to the lifestyle choices, climate change, industrialization of sectors, and typical regulation policies by power management sectors. The energy crisis arises due to two major problems: (1) the power supply and demand and (2) overconsumption of natural sources [1]. It is imminent to the present human generation and a serious issue for the future generation mankind. Alternative energy harvesting (AEH) options and energy policies are introduced across the world to overcome the energy crisis [2].

To drive any electronic device/sensor effectively depends on the following key factors such as interfacing circuit components, a processing unit, a sensing unit to measure/monitor various stimuli, and a battery source to power up the whole unit [3]. Among them, removing the toxic, complex wiring battery source and reducing the interfacing circuit components are the prime factors to improve the smart electronic device technology. This is possible only by the development of a nano/micro-sustainable independent power source coming from the surrounding environment. The AEH approaches will have many benefits such as saving natural resources, eco-friendly operation, portable/wearable, low cost, and finally removing the battery energy.

Many researchers believed and developed that the AEH approaches using the multifunctional nanomaterials and its devices will reduce the partial amount of energy crisis and make it possible to create a new type of multifunction smart sensors/devices in various fields such as biomedical, automotive, wireless-sensor networks, and communication electronics [4]. The rapid development, the invention of new nanomaterials, and cost-effective device designs are highly desirable to utilize the surrounding waste mechanical energy (machine vibrations, human body motions, wind/water motions, and ocean waves) in our surrounding environment [5].

Until now, piezoelectric nanogenerators (PNGs) [6], triboelectric electric nanogenerators (TNGs) [7], pyroelectric nanogenerators (PyNG) [8], and thermoelectric generator (TMNG) [9] are widely investigated and practically demonstrated to convert the waste mechanical/thermal energy into useful electrical energy. Among them, PNG technology is highly reliable, stable operations, smaller device area, and diverse application fields. To date, extensive fabrication methods, growth of various one-dimensional (1D)/two-dimensional (2D) inorganic piezoelectric nanostructures (NSs) on plastic substrates [6], flexible piezoelectric polymer films, and device designs (planar, stretchable, cylindrical, or fiber) were developed to improve the PNG technology as a prominent energy-harnessing approach for creating the sustainable independent power source to drive the low-power consumed electronic devices/sensors. Moreover, the device compatibility, electrical output performance $\left(\mathrm{nW} / \mathrm{cm}^{2}\right.$ to $\left.\mu W / \mathrm{cm}^{2}\right)$ under various harsh environments, and flexibility issues were optimized to think about the real-time commercialized PNG product [10]. On the other side, few PNGs have dual functionality such as wearable/portable independent power source to drive the commercial electronic devices and can also work as a self-powered sensor (or a battery-free sensor) to measure/monitor the various physical, chemical, biological, and optical stimuli [11]. The study reports suggest that PNG technology based on the type of materials is classified into three categories such as (1) inorganic 
nanostructures (NSs)-based PNG, (2) polymer matrix-based PNG, and (3) composite (polymer + inorganic NPs) PNG as shown in Figure 1 [12]. Wang et al. reported the first PNG reportbased $\mathrm{ZnO}$ nanostructures (named as inorganic PNG) and later showed the many possible ways to improve the instantaneous power density of the PNG [6]. Many other fellow researchers across the world extend the growth of other nanostructures and its device designs for inorganic PNG, but it has few ample drawbacks such as typical growth process of piezoelectric NSs, brittleness, lower force limits, failure instability, and leakage current issues [6, 10, 11]. Further, PNG was implemented with the flexible polyvinylidene fluoride (PVDF) and its copolymers due to its high flexibility, easy process to prepare flexible films, low electrical output performance, and accepting large mechanical force. However, it has one major disadvantage such as low piezoelectric coefficient and relative permittivity at room temperature than the inorganic

Drawbacks

Growth of nanostructures

Leakage current issue

Low force limit

Brittleness
Drawbacks

Low $d_{33}$ and $\varepsilon$ at $R T$

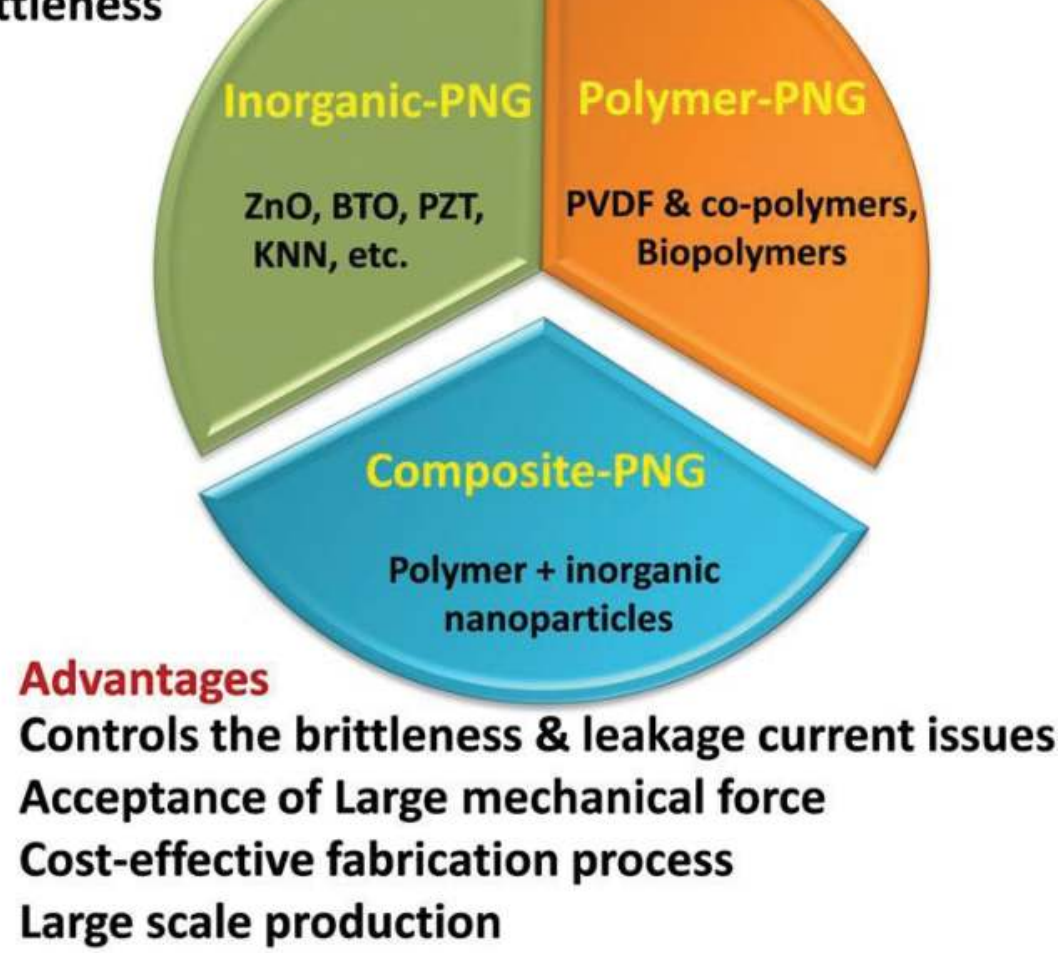

Figure 1. The schematic represents the overview of PNGs: classification, drawbacks, and advantages. 
piezoelectric NSs. Recently, our group and many other research groups successfully overcome the issues of the inorganic and polymer PNGs by developing the composite PNG technology [13-15]. The major key factor in designing the efficient, flexible composite PNG device is the development of multifunctional hybrid or composite piezoelectric structures (HPSs). It indirectly depends on the selection of individual high-performance inorganic and polymer materials and cost-effective fabrications processes. These kinds of composite PNGs are highly suitable to work as sustainable independent power sources as well as self-powered sensors to measure various physical parameters such as physical, optical, biological, and chemical stimuli.

The chapter describes the cost-effective HPSs (flat, spherical beads, worm structures, micropillars, irregular films, and hemispherical strips) developed by the simple fabrication processes such as solution-casting technique (SCT) [13, 14], ionotropic gelation process (IGT) [15], and groove technique (GT) [16]. The digital photographs of the as-fabricated HPSs are shown in Figure 2a. The HPSs were fabricated using the particular weight ratio of the
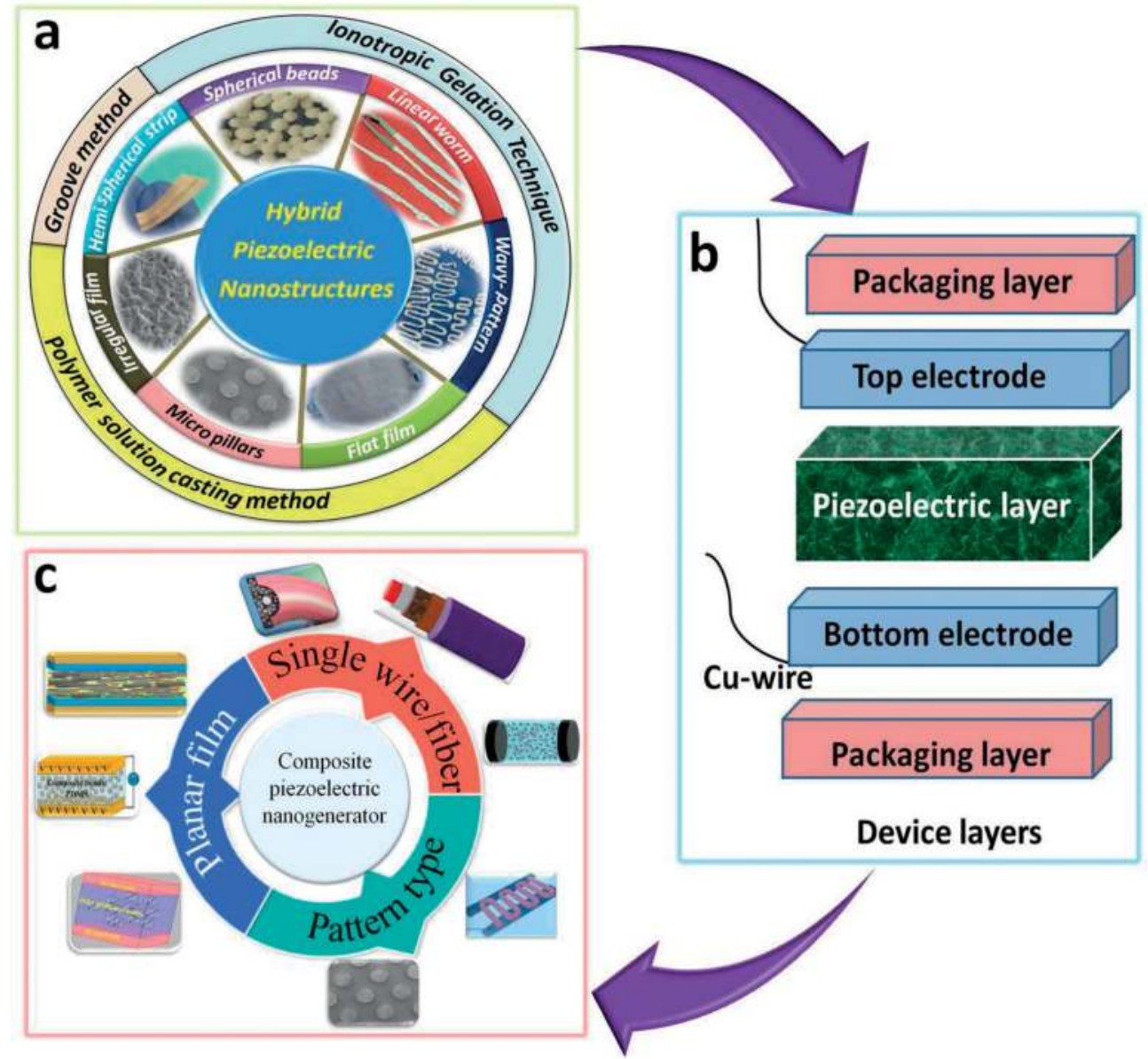

Figure 2. (a) The schematic represents the proposed HPSs using the polymer SCT, ITG, and GT. (b) Device layers of the composite PNG. (c) Possible types of composite PNGs such as planar film, pattern type, and single wire/fiber. 
inorganic nanostructures such as $\mathrm{BaTiO}_{3}$ nanoparticles and nanocubes, and the polymers are PVDF (polydimethylsiloxane), PDMS, and calcium alginate biopolymer. Further, these structures are used to fabricate the composite PNG devices. The device structures have three main layers such as (1) piezoelectric active layer (i.e., HPSs) and (2) top/bottom electrode layers (aluminum $(\mathrm{Al})$, gold $(\mathrm{Au})$, and platinum $(\mathrm{Pt})$ ) on the HPSs and top/bottom packaging layers (PDMS) as shown in Figure $\mathbf{2 b}$. The sandwich structure of all these layers without an airgap forms as a composite PNG device. Based on our previous works, the composite PNGs classified as three types such as planar film, pattern type, and single wire/fiber-type PNGs, and the corresponding schematic diagrams are shown in Figure 2c. Further, these PNGs are used as self-powered sensors/systems to monitor the fluid velocity and $\mathrm{pH}$ value of the alkaline solution. Self-powered flexion sensor to classify/measure the individual finger motions, nonlinear muscle motions of human body parts, acceleration sensors to measure the various accelerations of the linear motor shaft, and driving the commercial light-emitting diodes (LEDs)/liquid crystal display (LCD).

\section{Experimental section}

The hybrid or composite piezoelectric structures (HPSs) were developed using the homogeneous mixture of the various inorganic nanostructures, fillers into the polymer matrix. The shapes of the HPSs mainly depended upon the selection of the template polymer matrix, fabrication method, and the type of raw materials used (particular amount). In this case, the developed HPSs (flat film, spherical beads, linear and nonlinear worms, and hemispherical strip) are having multifunctional properties, and the process is eco-friendly, cost-effective, and easy to make the large-scale production at a given time interval. The followed synthesis procedures for functional nanoparticles and the fabrication methods for HPSs are solid-state reaction (SSR) nanoparticles [15], molten salt method (MSM) nanocubes [13], solution-casting technique (SCT)-flat film [13], ionotropic gelation process (ITG) spherical beads [15], worm structures [17, 18], and groove technique (GT)hemispherical strip [16].

\subsection{Synthesis of $\mathrm{BaTiO}_{3}$ nanoparticles and $\mathrm{BaTi}_{(1-x)} \mathrm{Zr}_{x} \mathrm{O}_{3}$ nanocubes}

\subsection{1. $\mathrm{BaTiO}_{3}$ nanoparticles (BTO NPs)}

The crystalline, randomly oriented BTO NPs were synthesized by SSR method [15], and the corresponding possible chemical reaction is.

$$
\mathrm{BaCO}_{3} \text { (solid) }+\mathrm{TiO}_{2} \text { (solid) } \leftrightarrow \mathrm{BaTiO}_{3} \text { (solid) }+\mathrm{CO}_{2} \text { (gas) }
$$

Here, the precursor materials such as barium carbonate and titanium dioxide were taken according to the atomic ratio of the final product and thoroughly grinded in the ethanol medium. The obtained homogeneous powder was placed in an alumina tube and fired at an elevated temperature of $1200^{\circ} \mathrm{C}$ for $2 \mathrm{~h}$ in an open tube furnace. After cooling down to the room temperature, the alumina tube was removed from the furnace and the final product collected and crushed into a fine powder form. 


\subsection{2. $\mathrm{BaTi}_{(1-x)} \mathrm{Zr}_{x} \mathrm{O}_{3}$ nanocubes (BTZO NCS)}

The MSM for synthesizing the inorganic perovskite nanostructures (NSs) [12] reduces the processing temperature and improves crystalline quality as compared to the NSs prepared by the SSR method. Here, the BTZO NCs are prepared by the homogeneous mixer of the reactant precursors $\left(\mathrm{BaCO}_{3^{\prime}}, \mathrm{TiO}_{2}, \mathrm{ZrO}_{2}\right.$ ), the eutectic products (molten salts are $\mathrm{NaCl}, \mathrm{KCl}$, and $\mathrm{Na}_{2} \mathrm{SO}_{4}$ ) and fired at a temperature of $750^{\circ} \mathrm{C}$ for $3 \mathrm{~h}$ in an open tube furnace. After natural cooling, the obtained product was washed several times to remove the existing unwanted salts precursors and finally heated at $60^{\circ} \mathrm{C}$ /overnight in a hot air oven. The final product was again crushed into a fine, smooth powder used to evaluate the structural, surface morphological, and functional characterization studies. In this case, the various amounts of zirconium ( $\mathrm{Zr}$ ) foreign atoms $(x=0,0.05,0.1,0.15$, and 0.2$)$ were doped into the B-site of the parent BTO lattice and the corresponding structural, energy harvesting capability was investigated thoroughly [12].

\subsection{Fabrication of hybrid piezoelectric flat film}

Various types of the hybrid piezoelectric flat (HPF) films were developed by the mixing of the particular weight ratio or volume percentages of the inorganic NSs into the fixed amount of the template polymer matrix. The performance, functionality of the as-fabricated HPF film depends upon the optimized weight ratio, the thickness of the film, and the homogeneous distribution of the NPs into the template polymer matrix. Here, PVDF/BTO NCs, PVDF/BTZO NCs [12], PDMS/BTO NCs [19], and PVDF/activated carbon (AC)-based HPF films [20] were fabricated and tested to harness the mechanical energy and to sense/monitor the physical, chemical, and optical stimuli.

\subsubsection{Fabrication of the PVDF/BTZO NCs, PDMS/BTO NCS HPF, and PVDF/activated carbon $(A C)$ films}

Highly flexible, cost-effective PVDF/BTZO and PVDF/BTO NCs HPF films [12] were fabricated using the simple ultrasonication method followed by the SCT. The HPF film was made up of the two key steps. One, the transparent, homogeneous PVDF solution was prepared by the sonication (approximately $1 \mathrm{~h}$ ) of the PVDF powder (1 gm) into an 8-mL mixed solution obtained from the solvents such as $\mathrm{N}, \mathrm{N}$-dimethylmethanamide (DMF) $(5 \mathrm{~mL}) /$ acetone $(3 \mathrm{~mL})$. During the sonication process, there is a possibility to generate the high temperature and the polymerization of the PVDF, the phase may be possible to vary. Therefore, a cold-water bath setup arranged around the glass vials contains the PVDF powder/solvents. A clear transparent solution was obtained when the PVDF powder completely dissolved in DMF/acetone solution. Second is the particular weight ratio ( 0.5 or 1 , etc.) of the as-synthesized BTO NCs were weighted, poured into the transparent PVDF solution, and the ultrasonication process continued for one more hour. For every $15 \mathrm{~min}$ of the sonication process, small mechanical agitation was performed in glass vials using the fine glass rod. There is a high chance that the clustered BTO NCs will settle at the bottom of the glass vial, and the generated ultrasound energy from the microtip probe will not dissolve these clustered BTO NCs into the PVDF solution. A clear non-transparent, whitish-colored hybrid solution was obtained after the $90 \%$ dissolution of the BTO NCs into a polymerized PVDF solution. The obtained whitish hybrid solution was removed from the sonication setup 
and cooled down to room temperature in an air atmosphere. After that, the hybrid solution was poured into fixed glass Petri dish and heat treated at $60^{\circ} \mathrm{C}$ overnight. Highly flexible PVDF/BTO NCs HPF film was obtained when the solution completely dried. Further, the structural and functional properties of the as-fabricated films (desired dimension) were investigated carefully. The fabrication process of the remaining HPF films such as PVDF/Zr-doped BTO NCs [12], PDMS/BTO NCs [19], and PVDF/AC [20] was similar and is explained in detail in our previous published reports.

\subsection{Fabrication of hybrid (BTO NPs/calcium alginate) piezoelectric spherical beads (HSB), linear and wavy-pattern worm (LW, WPW) structures}

The HSB [15], HLW [17], and HWPW [18] structures were fabricated using the eco-friendly, cost-effective ITG process. In this case, nontoxic polysaccharide, three-dimensional scaffoldstructured biopolymer was used as a template matrix to prepare the HPSs. The IGT process mainly consists of three major key steps. One, the preparation of the clear transparent sodium alginate (Na-alg) solution using the alginic acid sodium salt $(2 \mathrm{~g}$ ) obtained from the brown algae magnetically stirred $\left(50^{\circ} \mathrm{C}\right.$ for $2 \mathrm{~h}$ ) in particular amount of double distilled water $(100 \mathrm{~mL})$. Second is the preparation of the non-transparent hybrid (Na-alg/BTO NPs) solution by the homogeneous mixing of the as-synthesized BTO NPs into the obtained transparent Na-alg solution. The hybrid solution was thoroughly stirred magnetically at the elevated temperature of $70^{\circ} \mathrm{C}$ for $2 \mathrm{~h}$ without the sign of BTO agglomeration. The third is the gelation, replacement of sodium atoms in a hybrid solution by the calcium atoms to form as the ionic gelation of the BTO NPs/calcium alginate (BTO NPs/Ca-alg). Here, the substitution of the Ca-atoms is possible, when the squeezed hybrid (Na-alg/BTO NPs) solution is poured into a $1 \mathrm{wt} \%$ of calcium chloride $\left(\mathrm{CaCl}_{2}\right)$ bath. The procedure mentioned above is common for three structures such as HSB, HLW, and HWPW, and the only difference is the instrument used to drop the hybrid solution into the $\mathrm{CaCl}_{2}$ bath, free-fall height between the droplet position, and $\mathrm{CaCl}_{2}$ solution. The hybrid solution-loaded pipette dropper or a perforated metal mesh is used for HSB structure, the plastic syringe used for HLW structure, and the freehand drawing of the hybrid solution-loaded pipette dropper used for HWPW. The pure (Ca-alg) spherical beads and HLW structures were prepared by following the same protocol, whereas the pure wavy-pattern worm structures are not possible to prepare due to the high volatility of the Na-alg solution and the drawn pattern completely overlapped each other. Further, the structural, optical, and functional properties of the as-fabricated HPSs were investigated carefully $[15,17,18]$.

\subsection{Fabrication of hybrid (BTO NPs/PDMS) piezoelectric hemispherical strip}

The adaptable, highly flexible hybrid piezoelectric hemispherical strip (HPHS) is fabricated using the innovative, cost-effective GT. This kind of structure, approach is highly suitable to prepare the unconventional HPSs for measuring the nonlinear mechanical motions. Here, the shapes of the piezoelectric strip mainly depend on the type of the groove used during the fabrication time. The GT for HPHS mainly has four key steps. One, the preparation of the hemispherical groove on PET sheet and the placing of the flexible metal wire (titanium (Ti) 
or copper $(\mathrm{Cu})$ ) exactly at the center of the groove by using the two rigid supports at the ends of the PET groove. Second is the preparation of the hybrid (PDMS/BTO NPs) solution, pouring the required solution into that PET groove. Third is placing the loaded PET groove into a hot air oven and fired at $70^{\circ} \mathrm{C}$ for $30 \mathrm{~min}$. Fourth is the peel-off dried hybrid strip having one rectangular side base and another hemispherical side groove (replica of the PET groove) [16]. The flexibility, twisting capability, structural, functional, and energy-harvesting capability were evaluated and tested to monitor the human body physical moments.

Further, as fabricated, all the HPSs were utilized to harness the mechanical energy by fabricating the flexible/non-flexible PNGs and showed the possible prototype designs to monitor/sense the applied input stimuli without using the additional battery source, circuit components.

\section{Results and discussion}

The piezoelectric BTO and BTZO NCs prepared by the low-temperature MSM and the corresponding FE-SEM images at $200 \mathrm{~nm}$ are shown in Figure 3a and $\mathbf{b}$. The Raman spectra of NCs have the active vibration modes. They are [A1(TO), E(LO)] at $196 \mathrm{~cm}^{-1}, \mathrm{~A} 1$ (TO) at $256 \mathrm{~cm}^{-1}$, [B1(TO+LO), E] at $310 \mathrm{~cm}^{-1}$, and [A1(TO), E] at $520 \mathrm{~cm}^{-1}$, and $\left.\mathrm{A} 1(\mathrm{LO}), \mathrm{E}\right]$ at $720 \mathrm{~cm}^{-1}$. The major peak at $310 \mathrm{~cm}^{-1}$ confirms the existence of the tetragonal phase of BTO having the possibility of a higher piezoelectric coefficient than all other phases of BTO. Also, the crystalline phase confirmation of BTZO NCs, HPF films, was confirmed by the XRD patterns, FT-IR techniques, and the information was given in our previous published report [13]. As shown in Figure $\mathbf{2 b}$, the composite PNG was fabricated using the as-developed HPF film $(2.5 \mathrm{~cm} \times 2.5 \mathrm{~cm})$, top/ bottom electrodes and top/bottom packaging layers, respectively.

Initially, the mechanical force $11 \mathrm{~N}$ was applied exactly perpendicular to the PNG0 [PVDF/ BTO NCs] and PNG1 [PVDF/BTZO NCs $(x=0.1)$ ] devices, and the corresponding electrical responses are shown in Figure $3 \mathbf{e}$ and $\mathbf{f}$. Here, the PNG1 shows a higher electrical response $(11.9 \mathrm{~V}, 1.35 \mu \mathrm{A})$ than the PNG0 response $(7.99 \mathrm{~V}, 1.01 \mu \mathrm{A})$ due to the $\mathrm{Zr}$-doped BTO NCs. Here, the PVDF solution amount, device area, and applied force were kept constant. And the only difference is the substitution of a particular amount of $\mathrm{Zr}^{+4}$ into the $\mathrm{Ti}^{+4}$ site in the BTO lattice, which will be possible to enhance the piezoelectric coefficient than the pure BTO NCs. Here, the PVDF polymer has dual functionality such as supporting crosslinker to hold the BTO NCs to form a hybrid film and the small contribution to enhancing the generated electrical energy due to the small piezoelectric coefficient of PVDF ( $\beta$-phase). Further, the PNG1 was tested to measure the various fluid velocities of the water at the outlet of pipe, and the corresponding electrical responses are shown in Figure 4a. It demonstrates that the increasing water velocities $(31.43,78.6$, and $125.7 \mathrm{~m} / \mathrm{s}$ ) at the outlet pipe will be possible to generate different mechanical forces that act on the PNG1 and to respond with the increased piezoelectric potentials (self-powered fluid velocity sensor). It suggests that the PNG1 is a potential candidate to work as self-powered fluid velocity sensor without using any external battery energy and the additional circuit components. 

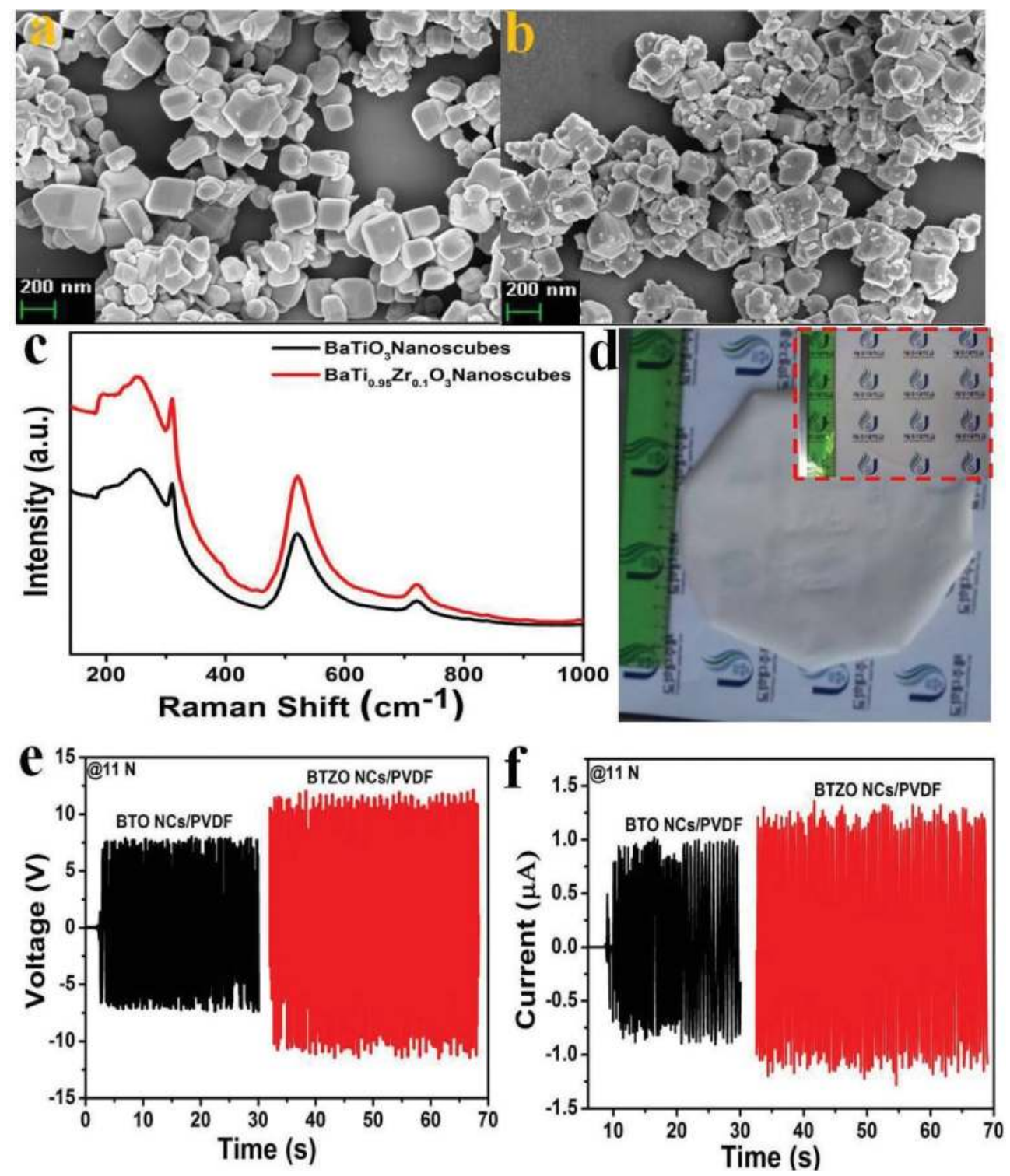

Figure 3. Flexible HPF film fabricated by solution-casting technique and its electrical response of PNGs. (a and b) High crystalline BTO and BTZO ( $x=0.1)$ NCs synthesized by the MSM. (c) Raman spectra of the BTO and BTZO NCs. (d) Digital photograph of the as-fabricated non-transparent HPF film and the inset shows the as-fabricated transparent pure PVDF film. (e and f) Generated electrical response (voltage, current) of the PNG0 (BTO NCs/PVDF) and PNG1 (BTZO $\mathrm{NCs} / \mathrm{PVDF})$ upon mechanical force $(11 \mathrm{~N})$. The figures are reproduced with the permission from Ref. [13]. Copyright of American Chemical Society (ACS) publications. 

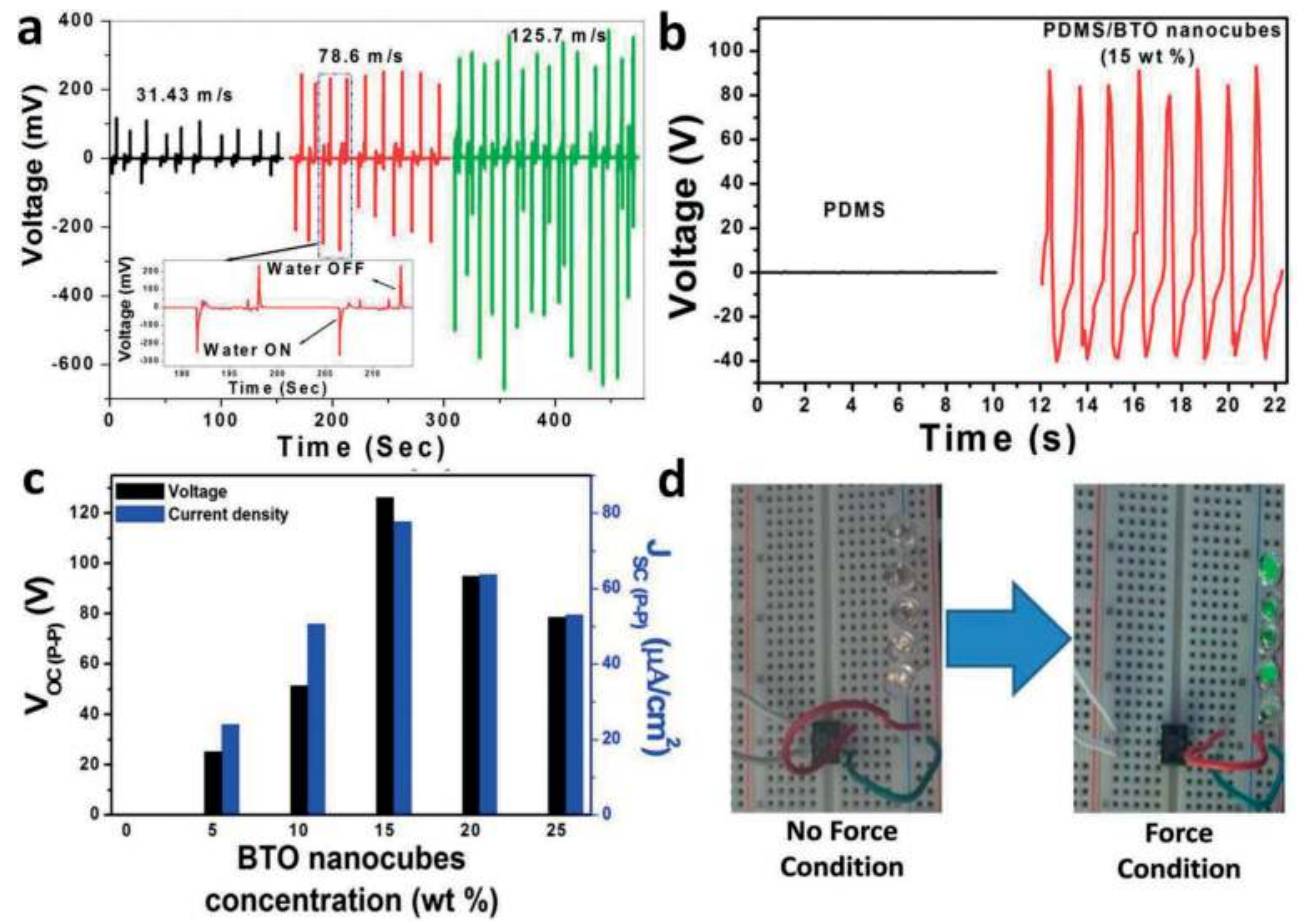

No Force

Force

Condition

Condition

Figure 4. (a) Demonstration of the self-powered fluid velocity sensor using the PNG1. (b) Output voltage response of PDMS and PDMS/BTO NCs-based devices upon the mechanical force. (c) PNG device electrical responses as a function of the weight ratio of BTO NCs into the PDMS matrix. (d) Driving commercial five green LEDs using the PDMS/BTO NCs film-based PNG device (15 wt\%). The figures are reproduced with the permission from Refs. [13, 19]. Copyright of American Chemical Society (ACS) publications.

Similarly, the PNG fabricated with the HPF film is made up of the flexible PDMS/BTO NCs (thickness $\approx 790 \mu \mathrm{m}$ ), and the corresponding electrical responses are shown in Figure $4 \mathbf{b}$ and $\mathbf{c}$. The PNG device with $15 \mathrm{wt} \%$ of BTO NCs generates a higher amount of piezoelectric potential electrical energy upon a low mechanical force of 988.14 Pa. Whereas in the case of the PNG device without BTO NCs, it generates almost zero electrical output upon mechanical force, because PDMS matrix does not have any piezoelectric property (non-centrosymmetric behavior) as shown in Figure $\mathbf{4 b}$. Also, the PNG electrical output upon constant mechanical force as a function of the weight ratio of BTO NCs into the PDMS has been verified, and the corresponding electrical response is shown in Figure 4c. It demonstrates that the linear increment output behavior is observed when increasing the weight ratio of BTO NCs (i.e., from 0 to $15 \mathrm{wt} \%$ ) in PDMS matrix. Beyond the $15 \mathrm{wt} \%$ of BTO NCs into the PDMS, it will result in the decreased electrical output. It may be due to the agglomeration of BTO NCs in the PDMS results in the lower cumulative electric dipole moment, and at the same time, the applied mechanical force may not be possible to distribute evenly on the PNG surface. Next, the potentiality of the PNG device ( $15 \mathrm{wt} \%$ of BTO NCs) output was tested to drive the commercial purchased five green LEDs connected in series. LEDs were in OFF condition when no mechanical force acts on the 
PNG and successfully lit up when the force acts on the PNG device as shown in Figure $4 \mathrm{~d}$. It demonstrates that the as-developed HPF film-based device is a highly potential candidate to work as a sustainable independent power source.

Researchers are directed toward the development of the cost-effective, eco-friendly fabrication methods to prepare the innovative HPSs-based PNG devices for various applications. For the first time, ionotropic gelation (ITG) technique and 3D template structure-based alginate biopolymer were introduced into the energy-harvesting field, and the possible HPSs for harnessing the waste mechanical energy is shown. Figure 5 shows the digital photographs, the surface morphology of the as-fabricated HSB, HLW, and HWPW structures and the corresponding PNG device electrical responses. Here, the piezoelectric tetragonal crystalline phase of BTO NPs was not changed even after the substitution of BTO NPs into the Ca-alginate polymer. The HSB-based PNG device fabricated by mixing the particular amount of HSBs into the PDMS matrix forms a flat film, and the followed the above-demonstrated sandwich fabricating the structure. Figure 5a(iii) demonstrates that the higher electrical output voltage $(\leq 11 \mathrm{~V})$ generated for the HSB-based devices as compared to the pure PDMS $(<3 \mathrm{~V})$ and pure Ca-alg beads/ PDMS ( $\leq 7 \mathrm{~V}$ )-based devices upon constant mechanical force. Here, a small amount of electrical

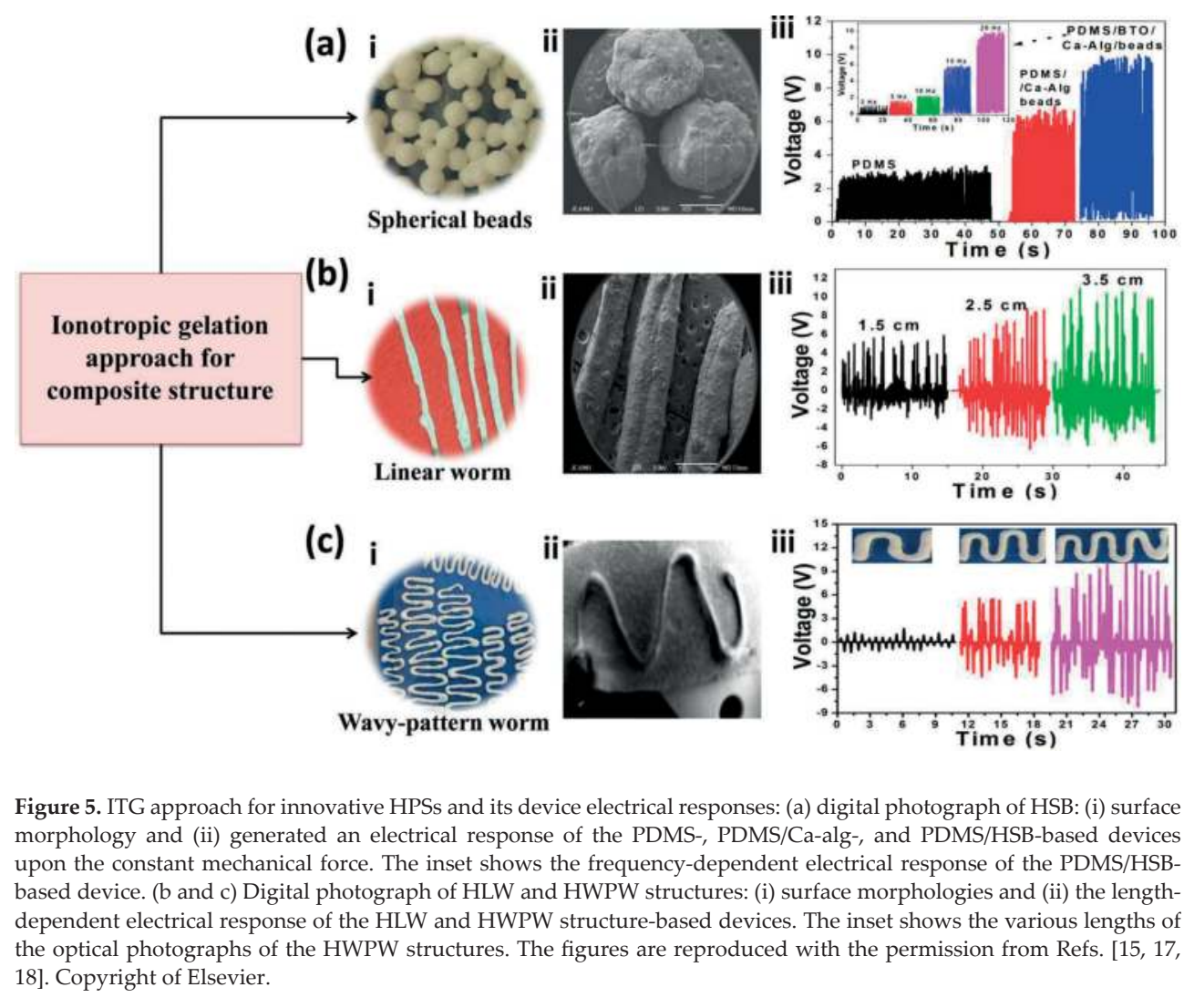


output voltage is generated when there is no BTO NCs in the beads and the PDMS matrix, due to the existence of the small air gap in the as-fabricated final device structure results in the introduction of the triboelectric effect of Ca-alg and PDMS layer along with the Al foil electrode. Therefore, the generated final electrical output of HSB-based PNG is due to the synergistic electric of the piezoelectric effect from the BTO NCs and triboelectric effect from the Ca-alg and PDMS layers. Further, the HLW and HWPW structure-based PNG devices were fabricated and tested to harness the mechanical energy. The generated HLW-based device positive output increased from $(\approx 5 \mathrm{~V})$ to $(\approx 10 \mathrm{~V})$ upon hand mechanical force, when the length of the HLW structure increased from 1.5 to $3.5 \mathrm{~cm}$ as shown in Figure $\mathbf{5 b}$ (iii). The similar behavior is observed for the HWPW-based PNG devices, that is, the voltage increased from $(\approx 1.5 \mathrm{~V})$ to $(\approx 10 \mathrm{~V})$, when the length of the HWPW structure is from 0.85 to $1.95 \mathrm{~cm}$ as shown in Figure $5 \mathrm{c}$ (iii). To identify the role of Ca-alg in these structures, the pure linear worm structure-based device (without an air gap) has been fabricated and the zero electrical output has been generated, which confirms that no triboelectric effect was introduced into these device structures. Further, these structurebased devices were used to measure the flexion/extension of the individual finger motions (human hand) and the cumulative fist movement, that is, self-powered flexion sensor (SFS) as shown in Figure $6 \mathbf{a}$ and $\mathbf{b}$. Also, the monitoring of the $\mathrm{pH}$ value of the alkaline solutions ranges from 12 to 8 (self-powered pH sensor (SPS)) as shown in Figure $6 \mathbf{c}$ and $\mathbf{d}$.
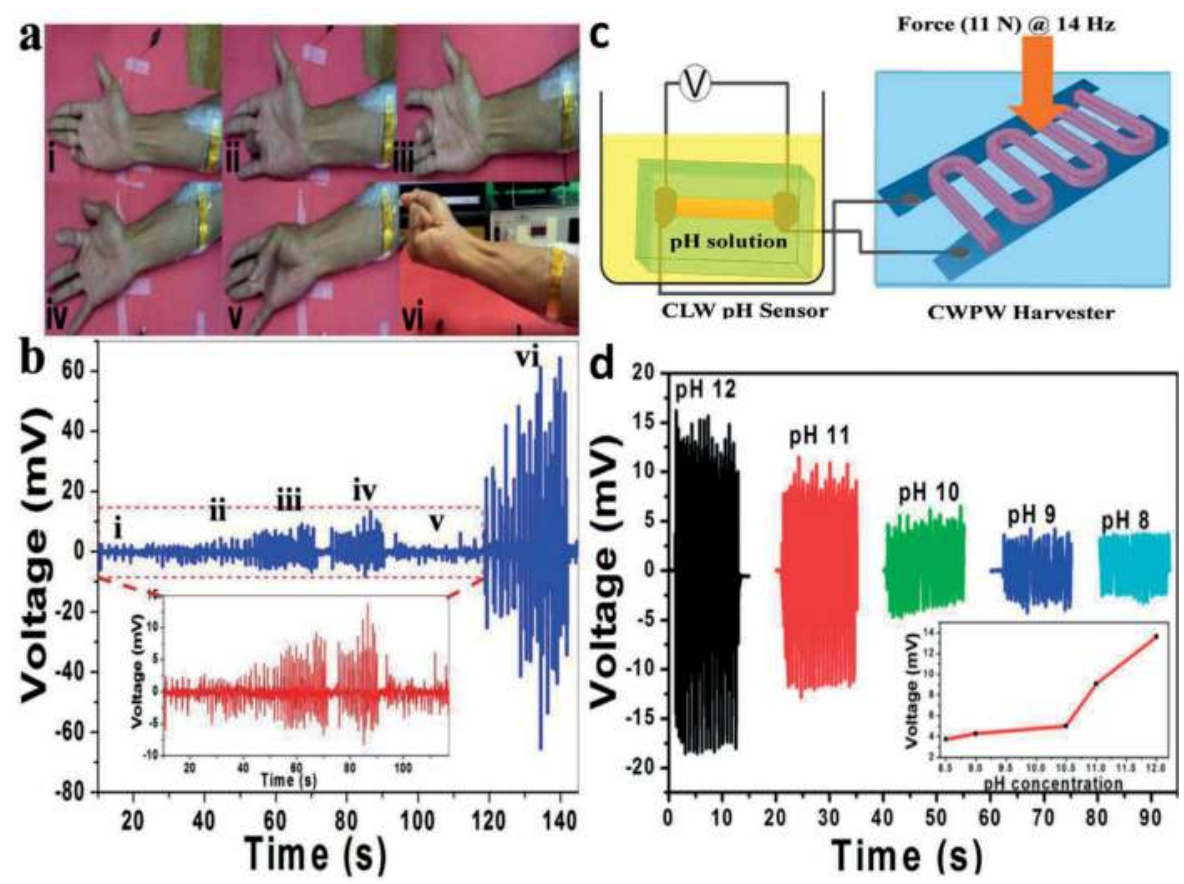

Figure 6. (a) Demonstration of the SFS to measure individual human finger flexion/movements and the strip-type HSBbased PNG device mounting on the forearm of the human hand. (b) The electrical response of the SFS concerning the finger motions and the inset shows the magnified electric response of SFS. (c) Schematic represents the demonstration of the SPS using the parallel connection between the HWPW-based PNG device and the HLW pH sensor. (d) The electrical response of the SPS under various alkaline solutions ranges from a value of 12 to 8 . The figures are reproduced with the permission from Refs. [17, 18]. Copyright of Elsevier. 
In the case of SFS, the finger motion generates the small amount of mechanical force through the tendon-muscle unit of the forearm, which perpendicularly acts on the SFS results in the change in electrical response concerning the individual finger. Here, the location of the SFS on the forearm, length, and sensitivity of the SFS will determine the perfect classification and measurement of the individual fingers (thumb, index, middle, ring, and pinky) as shown in Figure $\mathbf{6 b}$. Whereas in the case of the SPS, HWPW-based device works as a sustainable independent power source to drive the HLW pH sensor as shown in Figure 6c. The output voltage of the HLW pH sensor decreases from 33 to $7.3 \mathrm{mV}$, when the $\mathrm{pH}$ buffer solution changed from 12 to 8 . It indicates that the change in resistance of the $\mathrm{pH}$ sensor decreases gradually concerning the decrease in the $\mathrm{pH}$ value of buffer solution as shown in Figure $6 \mathrm{~d}$.

To measure the nonlinear surface motions of any object or biological body requires the highly adaptable, flexible, and having a small device area. For this, efficient, adaptable HPHS are fabricated by the invention of the cost-effective GT. Here, the hybrid strips are possible to make any shape depending on the type of the groove used, proper hybrid (polymer + inorganic $0.3 \mathrm{Ba}_{0.7} \mathrm{Ca}_{0.3} \mathrm{TiO}_{3}-0.7 \mathrm{BaSn}_{0.12} \mathrm{Ti}_{0.88} \mathrm{O}_{3}$ (BCST) NPs) solution. The synthesis process, crystalline phase information, ferroelectric hysteresis loop characterization, and the surface morphological studies of BCST NPS were given in our previous report [16]. Figure 7a shows the schematic diagram of the detailed process of the GT for the HPHS explained in the experimental section. The crystalline phase of the HPHS (PDMS/BCST NPs), pure PDMS, and BTO NPs was investigated by the Raman spectra as shown in Figure $7 \mathbf{b}$. Briefly describing, the peak
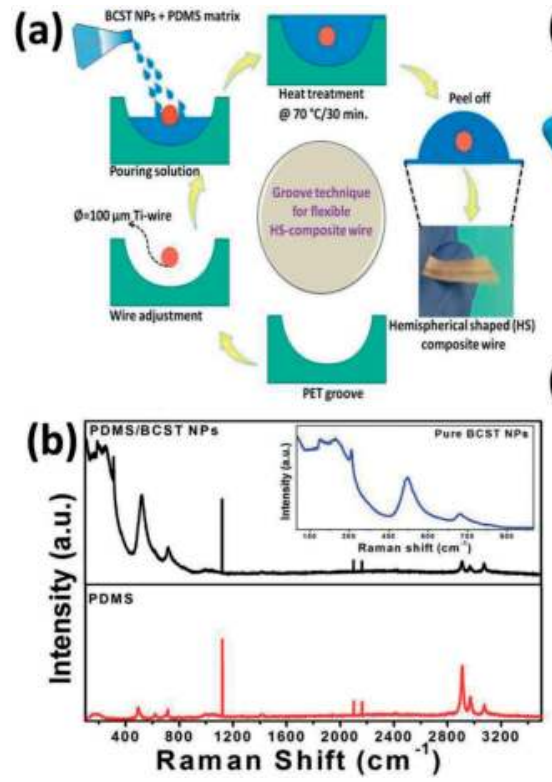

(c)

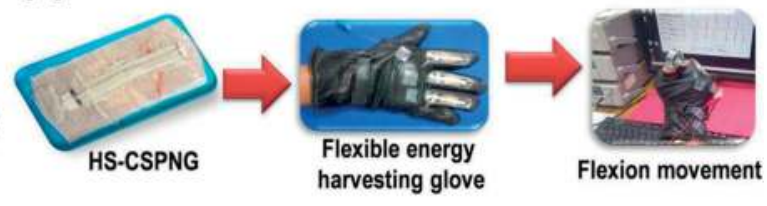

(d)

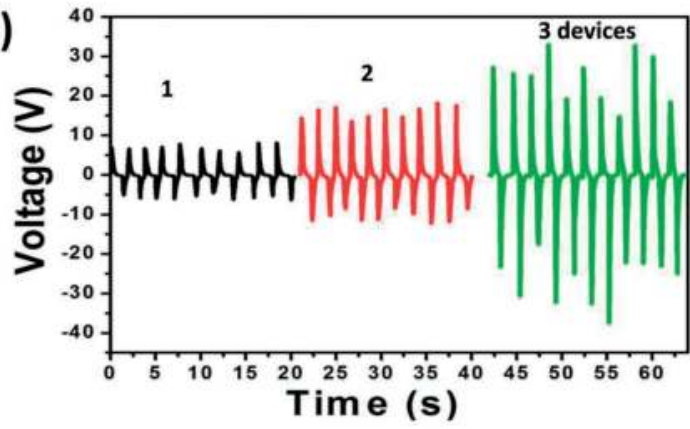

Figure 7. (a) Cost-effective grove technique (GT) for preparing the HPHS and its optical photograph. (b) Raman spectra of the pure PDMS, HPHS and the inset shows the high crystalline BTO NPs. (c) Optical photograph of the as-developed hemispherical composite strip-type PNG (HS-CSPNG) and the development of flexible energy-harvesting glove (using three devices) and its flexion movement during the electrical measurement. (d) Output voltage response of flexible energy harvesting glove upon the flexion/extension movements of human hand fingers. The figures are reproduced with the permission from Ref. [16]. Copyright of Royal Society of Chemistry. 
between 300 and $310 \mathrm{~cm}^{-1}$ in HPHS and BCST NPs was confirmed by the existence of the higher tetragonal phase content. Whereas the peaks in PDMS spectra confirm the existence of the $\mathrm{CH}_{3}$ (symmetric, asymmetric stretching's), $\mathrm{Si}-\mathrm{C}$ (symmetric), $\mathrm{Si}-\mathrm{CH}_{3}$ (symmetric), and $\mathrm{Si}-\mathrm{O}-\mathrm{Si}$ (symmetric) vibration bands. Further, as-developed HPHS are used to fabricate the HS-CSPNG devices, and flexible energy-harvesting glove and the corresponding digital photographs are shown in Figure 7c. The flexible energy-harvesting glove was tested to harness the waste biomechanical energy, that is, harnessing the flexion/extension movements of the finger converted into a useful electrical signal. The single HS-CSPNG device generates an approximately peak-to-peak voltage of $\approx 10 \mathrm{~V}$, a series connection of two HS-CSPNGs and three HS-CSPNGs generates $(\leq 30 \mathrm{~V})$ and $(\leq 60 \mathrm{~V})$ upon flexion/extension movements of the finger (Figure 7d). It demonstrates that the as-developed HPHSs and the HS-CSPNGs are highly potential candidates to harness the waste mechanical energy, having a reduced device area and low cost. Further, these HPHS are used to develop the self-powered muscle-monitoring system to measure the nonlinear surface motions of the human body parts at a time without using any additional storage battery, circuit components (reference). Other than these, as-developed PVDF/AC hybrid film was tested to harness the mechanical energy and also used to develop the self-powered acceleration sensor to measure the various accelerations of the linear motor shaft load. The detailed fabrication protocol, characterization analysis, and electrical responses were given in our previously published report [20]. The described basic approaches for piezoelectric nanogenerators and self-powered sensors in the present work can pave the way to develop the next-generation flexible/portable smart electronics/sensors.

\section{Conclusions}

In summary, the lead-free piezoelectric $\mathrm{BaTiO}_{3} \mathrm{NPs}, \mathrm{NCs}$, and BCST NPs along with the polymer support can be possible to generate the innovative HPSs to improve the instantaneous power density of the PNG. The flexible HPSs performance depends on the weight ratio of nanoparticles in a polymer matrix, thickness of the film, external poling process, and the applied perpendicular mechanical force. The fabrication methods for HPSs are cost-effective, eco-friendly, simple, and possible for the large-scale production. The synergistic (piezoelectric-triboelectric) effect at the material design level or the device design level will enhance the generated power density of the device. Few times, as-fabricated PNGs have a dual functionality such as harnessing the mechanical energy (sustainable independent power source) and can also work as a selfpowered sensor without using additional storage components. The results demonstrate that the HPSs and its nanogenerators are potential candidates to develop the self-powered sensors/systems to monitor the physical/optical/chemical stimuli and glow the low-power consumed LEDs.

\section{Acknowledgements}

This work was supported by the Jeju Sea Grant College Program 2017, funded by the Ministry of Oceans and Fisheries (MOF), and by the National Research Foundation of Korea (NRF), funded by the Korea Government Grant (2016R1A2B2013831). 


\section{Conflict of interest}

There are no conflicts to declare.

\section{Author details}

Nagamalleswara Rao Alluri ${ }^{1}$, Arunkumar Chanderashkear ${ }^{2}$ and Sang-Jae Kim²*

*Address all correspondence to: kimsangj@jejunu.ac.kr

1 Mechanical Engineering, Faculty of Applied Energy System, Jeju National University, Jeju Island, South Korea

2 Department of Mechatronics, Nanomaterials and Systems Lab, Jeju National University, Jeju Island, South Korea

\section{References}

[1] International Energy Outlook 2017. Available from: https://www.eia.gov/outlooks/ieo/ pdf/0484(2017).pdf [Accessed: 30-01-2018]

[2] Energy crisis, causes of the energy crisis and possible solutions of the energy crisis. Available from: https://www.conserve-energy-future.com/causes-and-solutions-to-theglobal-energy-crisis.php [Accessed: 30-01-2018]

[3] Yuan-Ping L, Dejan V. Self-powered electronics for piezoelectric energy harvesting devices (chapter-14). In: Lallart M, editor. Small-Scale Energy Harvesting. London, United Kingdom: InTechopen Limited; 2012. ISBN: 978-953-51-0826-9. DOI: 10.5772/51211

[4] Wang ZL, Wu W. Nanotechnology enabled energy harvesting for self-powered micro/ nanosystems. Angewandte Chemie, International Edition. 2012;51:2-24. DOI: 10.1002/ anie. 201201656

[5] Venkateswaran V, Nagamalleswara Rao A, Yuvasree P, Arunkumar C, Sang-Jae K. A flexible, planar energy harvesting device for scavenging roadside waste mechanical energy via the synergistic piezoelectric response of $\mathrm{K}_{0.5} \mathrm{Na}_{0.5} \mathrm{NbO}_{3}-\mathrm{BaTiO}_{3} / \mathrm{PVDF}$ composite films. Nanoscale. 2017;9:15122-15130. DOI: 10.1039/C7NR04115B

[6] Wang ZL, Song J. Piezoelectric nanogenerators based on zinc oxide nanowire arrays. Science. 2006;312:242-246. DOI: 10.1126/science.1124005

[7] Arunkumar C, Nagamalleswara Rao A, Saravanakumar B, Sophia S, Sang-Jae K. Human interactive triboelectric nanogenerator as a self-powered smart seat. ACS Applied Materials \& Interfaces. 2016;8:9692-9699. DOI: 10.1021/acsami.6b00548

[8] Ya Y, Wenxi G, Ken CP, Guang Z, Yusheng Z, Yan Z, Youfan H, Long I, Wang ZL. Pyroelectric nanogenerators for harvesting thermal energy. Nanoscale. 2012;12:28332838. DOI: 10.1021/nl3003039 
[9] Ya Y, Ken CP, Qingshen J, Jyh Ming W, Fang Z, Yusheng Z, Yue Z, Wang ZL. thermoelectric nanogenerators based on single Sb-doped ZnO micro/nanobelts. ACS Nano. 2012; 6:6984-6989. DOI: 10.1021/nn302481p

[10] Joe B, Steve D. Piezoelectric nanogenerators-A review of nanostructured piezoelectric energy harvesters. Nano. 2015;14:15-29. DOI: 10.1016/j.nanoen.2014.11.059

[11] Qiang Z, Bojing S, Li Z, Wang ZL. Recent progress on piezoelectric and triboelectric energy harvesters in biomedical systems. Advancement of Science. 2017;4:1700029. DOI: 10.1002/advs.201700029

[12] Kwi-Il P, Chang KJ, Na Kyung K, Lee KJ. Stretchable piezoelectric nanocomposite generator. Nano Convergence. 2016;3:1-12. DOI: 10.1186/s40580-016-0072-z

[13] Nagamalleswara Rao A, Saravanakumar B, Sang-Jae K. Flexible, hybrid piezoelectric film $\left(\mathrm{BaTi}_{(1-x)} \mathrm{Zr}_{x} \mathrm{O}_{3}\right) /$ PVDF nanogenerator as a self-powered fluid velocity sensor. ACS Applied Materials \& Interfaces. 2015;7:9831-9840. DOI: 10.1021/acsami.5b01760

[14] Saravanakumar B, Thiyagarajan K, Nagamalleswara Rao A, Shin SY, Taehyun K, Lin ZH, Sang-Jae K. Fabrication of an eco-friendly composite nanogenerator for self-powered photosensor applications. Carbon. 2015;84:56-65. DOI: 10.1016/j.carbon.2014.11.041

[15] Nagamalleswara Rao A, Sophia S, Arunkumar C, Saravanakumar B, Jeong JH, SangJae K. Piezoelectric $\mathrm{BaTiO}_{3}$ /aginate spherical composite beads for energy harvesting and self-powered wearable flexion sensor. Composites Science and Technology. 2017;142:6578. DOI: 10.1016/j.compscitech.2017.02.001

[16] Nagamalleswara Rao A, Venkateswaran V, Arunkumar C, Saravanakumar B, Sang-Jae K. Adaptable piezoelectric hemispherical composite strips using a scalable groove technique for a self-powered muscle monitoring system. Nanoscale. 2018;10:907-913. DOI: 10.1039/C7NR06674K

[17] Nagamalleswara Rao A, Sophia S, Arunkumar C, Saravanakumar B, Lee GM, Jeong JH, Sang-Jae K. Worm structure piezoelectric energy harvester using ionotropic gelation of barium titanate-calcium alginate composite. Energy. 2017;118:1146-1155. DOI: 10.1016/j. energy.2016.10.143

[18] Nagamalleswara Rao A, Sophia S, Arunkumar C, Saravanakumar B, Jeong JH, Sang-Jae K. Self-powered $\mathrm{pH}$ sensor using piezoelectric composite worm structures derived by ionotropic gelation approach. Sensors and Actuators B: Chemical. 2016;237:534-544. DOI: 10.1016/j. snb.2016.06.134

[19] Nagamalleswara Rao A, Arunkumar C, Venkateswaran V, Yuvasree P, Sophia S, Ji Hyun J, Sang-Jae K. Scavenging biomechanical energy using high-performance, flexible $\mathrm{BaTiO}_{3}$ nanocube/PDMS composite films. ACS Sustainable Chemistry \& Engineering. 2017;5:47304738. DOI: 10.1021/acssuschemeng.7b00117

[20] Nagamalleswara Rao A, Arunkumar C, Ji Hyun J, Sang-Jae K. Enhanced electroactive b-phase of the sonication-process-derived PVDF-activated carbon composite film for efficient energy conversion and a battery-free acceleration sensor. Journal of Materials Chemistry C. 2017;5:4833-4844. DOI: 10.1039/c7tc00568g 